\title{
Li Diffusion in All-Solid-State Batteries Imaged Through Optical and Electron Transparent Electrodes
}

\author{
Alexander Yulaev ${ }^{1,2,3}$, A. Alec Talin ${ }^{4}$, Marina S. Leite ${ }^{2,5}$ and Andrei Kolmakov ${ }^{1}$ \\ 1. Center for Nanoscale Science and Technology, NIST, Gaithersburg, MD, USA. \\ 2. Department of Materials Science and Engineering, University of Maryland, College Park, MD, USA. \\ ${ }^{3 .}$ Maryland NanoCenter, University of Maryland, College Park, MD, USA. \\ 4. Sandia National Laboratories, Livermore, CA, USA \\ ${ }^{5 .}$ Institute for Research in Electronics and Applied Physics, University of Maryland, College Park, USA.
}

The development of all-solid-state batteries has opened new scientific and engineering perspectives towards miniature standalone micro-electronics and medical devices [1]. All-solid-state batteries with thin-film solid electrolytes exhibit a high power-to-weight ratio and double or even triple energy density of liquid electrolyte batteries. In addition, solid batteries enable significant reduction of safety risks related to thermal runaway observed for liquid organic electrolytes [2]. Despite growing interest in the energy storage community dedicated to all-solid-state thin-film batteries, the electrochemical and electromechanical interfacial processes remain largely unexplored at the nanoscale. In particular, LiPON (lithium phosphorus oxynitride) is successfully used as a solid electrolyte for Li-ion batteries [3]. However, the factors that control the intercalation and homogeneity of Li transport in the solid electrolyte are still unknown. Balke et al. utilized electrochemical strain microscopy to study Li-ion transport through LiPON into Si anode at nanoscale level [4]. Ruzmetov et al. employed transmission electron microscopy (TEM) to probe a nanowire battery with a radial geometry to reveal the scaling limit of a LiPON layer [5].

Here, we report spatially- and depth-resolved study of Li intercalation into a thin-film solid electrolyte applying a combination of optical and electron microscopy. Varying the depth of electron interaction volume by changing energy of electron beam from $2 \mathrm{keV}$ to $15 \mathrm{keV}$, we selectively probe an electrolyte lithiated on one side at different depths. This approach can be used to reconstruct 3D diffusion pathways of Li similar to scanning electron microscopy (SEM) tomography. Our model thin-film all-solid-state Li battery consists of ultrathin silicon anode, LiPON as electrolyte, and metallic lithium as the positive electrode (Fig. 1). To make such a stack, $430 \mathrm{~nm}$ thick LiPON was sputtered onto $35 \mathrm{~nm}$ thick Si freestanding membrane (Fig. 1a). Micro-patterned $100 \mathrm{~nm}$ thick nickel layer was deposited onto electrolyte by e-beam evaporation through a stencil mask to serve as a diffusion barrier for Li intercalation. Using $\mathrm{Ni}$ as a stencil mask, spatially separated metal Li patches were created on LiPON via thermal evaporation (Fig. 1b). Lithium diffusion in LiPON layer was monitored using SEM imaging through the $35 \mathrm{~nm}$ thick Si membrane. Due to difference in electron yield from pristine and lithiated LiPON regions, the Li transport in the electrolyte can be mapped using different electron beam accelerating voltages. Fig. $2 \mathrm{a}$ was recorded at $2 \mathrm{kV}$ electron beam and, therefore, shows $\mathrm{Li}$ distribution close to surface $(\approx 40 \mathrm{~nm})$ region. At higher accelerating voltage $(15 \mathrm{kV})$, the electron probing depth spans up to $1.9 \mu \mathrm{m}$; consequently, (Fig. 2b) SEM image depicts Li distribution in electrolyte next to Ni layer. Comparative SEM image analysis reveals the difference in Li spatial distribution via specific choice of electron energy level (Fig. 2c). Monte-Carlo simulations of electron beam interaction in LiPON at $\mathrm{E}_{b}=2 \mathrm{keV}$ and $15 \mathrm{keV}$ supported the observed results. In addition, due to optical transparency of thin-film electrolyte and ultrathin Si anode, Li diffusion profiles at both sides of the battery can be visualized optically (Fig. 2d). We envision that depth selective electron microscopy of thin-film ion batteries will help elucidate the role of temperature, chemical and morphological inhomogeneity of the electrolyte in Li ion transport [6]. 


\section{References:}

[1] J. Bates, et al., Solid State Ionics, vol. 135, 2000, pp. 33-45.

[2] R. Huggins, "Advanced batteries: materials science aspects", (Springer Science \& Business Media), 2008.

[3] J. Bates, et al., Journal of Power Sources, vol. 43, 1993, pp. 103-110.

[4] N. Balke, et al., Nano letters, vol. 10, 2010, pp. 3420-3425.

[5] D. Ruzmetov, et al., Nano letters, vol. 12, 2011, pp. 505-511.

[6] This work has been supported in part by the NIST-CNST/UMD-IREAP Cooperative Agreement.
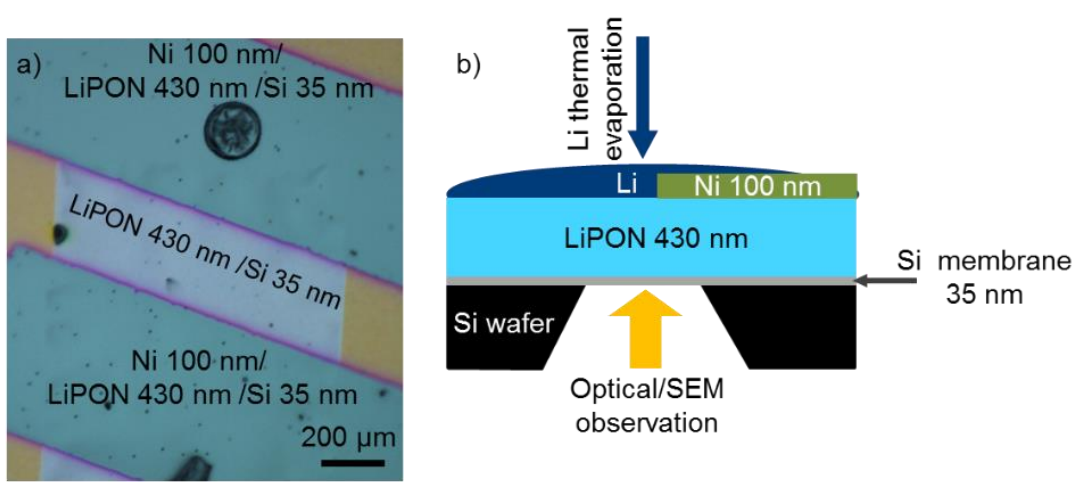

Figure 1. Sample structure: a) The optical image of the top side of the battery before Li evaporation; b) The schematics of the cross sectional view of the battery. Ultra-thin Si membrane is used as optical and electron transparent window.
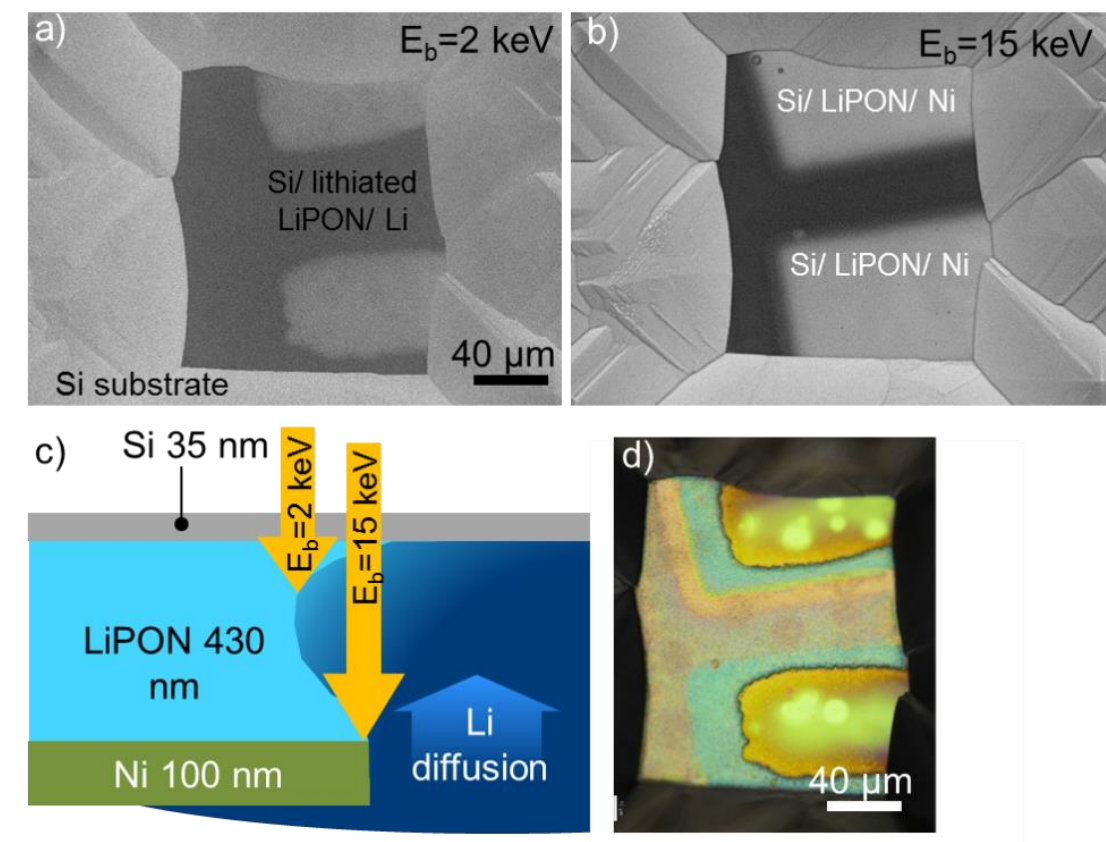

Figure 2. a), b) SEM images obtained at $E_{b}=2 \mathrm{keV}$ and $15 \mathrm{keV}$, respectively; bright areas in Si windows regions correspond to electron scattering from $\mathrm{Ni}$ electrode; dark regions represent electron yield from lithiated electrolyte; c) schematic explanation of SEM contrast formation at different energies of the electron beam; d) optical image: lithiated regions are seen through $35 \mathrm{~nm} \mathrm{Si}$ and LiPON layers. 